\title{
Summary of the CKM 2016 working group on high-energy flavor physics
}

\section{Veronique Boisvert*}

Department of Physics, Royal Holloway, University of London, Egham, TW20 OEX, UK

E-mail: Veronique.Boisverterhul.ac.uk

\section{Joachim Brod}

Fakultät für Physik, TU Dortmund, D-44221 Dortmund, Germany

E-mail: joachim.brodetu-dortmund.de

We summarize recent developments in single top quark and top-quark pair production, forwardbackward and charge asymmetries, constraints on top-quark FCNC, searches for CP violation, and the direct measurement of $V_{t b}$.

9th International Workshop on the CKM Unitarity Triangle

28 November - 3 December 2016

Tata Institute for Fundamental Research (TIFR), Mumbai, India

\footnotetext{
${ }^{*}$ Speaker.
} 


\section{Introduction}

The Higgs boson, and the top quark as the heaviest quark flavor, generally play a prominent role in theories of physics beyond the standard model, and thus complement the searches for new physics using precision observables in the $B, D$, and $K$ sector. The copious production of top quarks and Higgs bosons at the LHC provides a new window to search for new high-energy phenomena. In addition, precision probes like electric dipole moments and flavor observables give complementary constraints on new interactions.

In this short review, we discuss cross sections (Sec. 2), top-quark properties and new physics (Sec. 3), asymmetries (Sec. 4), the interplay between Higgs and top physics (Sec. 5), beyond standard-model (BSM) searches (Sec. 6), top-quark effective field theory (Sec. 7), and precision probes (Sec. 8).

\section{Single top and top quark pair production}

An indispensable ingredient in any search for new physics is a reliable standard model prediction. This applies also to top quark physics. The total top quark pair production cross section is known to next-to-next-to-next-leading order in the strong coupling, both for the gluon initial state [1] and the $q \bar{q}$ initial state [2] . The measurement of a large top quark forward-backward asymmetry at the Tevatron (see Sec. 4) was one of the motivations to extend these calculations also the differential cross sections, both for the LHC [3] and for the Tevatron [4].

These calculations also allowed for a global fit of the large- $x$ gluon PDF for at NNLO, which is an important input for the prediction of heavy particle production. These issues, together with theoretical uncertainties, are discussed in more detail in [5].

The decay channels of $t \bar{t}$ production are labelled according to the $W$ boson decay channels. Not including the $\tau$ lepton decays, the smallest contribution to the $t \bar{t}$ production comes from the dilepton channel ( $\sim 5 \%)$ where both $W$ bosons decayed into leptons, the next contribution comes from the lepton+jets channel $(\sim 30 \%)$ where one of the $W$ boson decayed into leptons while the other decayed into hadrons, and the largest contribution $(\sim 44 \%)$ comes from the case where both $W$ bosons decayed into hadrons and is called the all-hadronic channel.

\subsection{Experimental results on $t \bar{t}$ production results}

The ATLAS and CMS experiments have measured the $t \bar{t}$ production cross-section at the centreof-mass energies of $7 \mathrm{TeV}, 8 \mathrm{TeV}$ and $13 \mathrm{TeV}$ (CMS also has a measurement at $5.02 \mathrm{TeV}$ ). In all cases, the measurements are compatible with the QCD prediction. At a centre-of-mass energy of $13 \mathrm{TeV}$, the most precise measurements are obtained using the dilepton channel where exactly one electron and one muon of opposite sign are requested. The ATLAS experiment makes use of events containing exactly one jet identified as coming from a $b$-hadron (b-tagged) as well as events containing exactly two b-tagged jets in order to simultaneously measure the $t \bar{t}$ production crosssection as well as the b-tagging efficiency. The CMS measurement requests at least two jets and at least one b-tagged jet. The respective results are given in table 1. The LHCb experiment has also recently measured the inclusive $t \bar{t}$ production cross-section using the $8 \mathrm{TeV}$ dataset [6]. 


\begin{tabular}{ll}
\hline \hline ATLAS & $\sigma_{t \bar{t}}=818 \pm 8$ (stat.) \pm 27 (syst.) \pm 19 (lumi.) $\mathrm{pb}$ \\
& $\varepsilon_{b}=0.559 \pm 0.004$ (stat.) \pm 0.003 (syst.) \\
\hline CMS & $\sigma_{t \bar{t}}=792 \pm 8$ (stat.) \pm 37 (syst.) \pm 21 (lumi.) $\mathrm{pb}$ \\
\hline theory & $\sigma_{t \bar{t}}=832_{-51}^{+46}$ (scale, $\mathrm{PDF}, \alpha_{s}$ ) pb \\
\hline \hline
\end{tabular}

Table 1: Inclusive $t \bar{t}$ production cross-section measurements using the dilepton channel at $13 \mathrm{TeV}$. An additional uncertainty of about $1.5 \%$ is present to account for the uncertainty in the beam energy at the LHC. The theoretical prediction is evaluated using the Top++2.0 program [7]. The calculation includes next-tonext-to-leading-order (NNLO) QCD corrections and resums next-to-next-to-leading logarithmic (NNLL) soft gluon terms $[2,1,8,9,10,11]$.

\begin{tabular}{ll}
\hline \hline ATLAS & $\sigma_{t \overline{t Z}}=0.92 \pm 0.29$ (stat.) \pm 0.10 (syst.) pb \\
& $\sigma_{t \bar{t} W}=1.50 \pm 0.72$ (stat.) \pm 0.33 (syst.) $\mathrm{pb}$ \\
\hline CMS & $\sigma_{t \bar{t} Z}=0.7_{-0.15}^{+0.16}$ (stat.) ${ }_{-0.12}^{+0.14}$ (syst.) pb \\
& $\sigma_{t \bar{t} W}=0.98_{-0.22}^{+0.23}$ (stat.) ${ }_{-0.18}^{+0.22}$ (syst.) pb \\
\hline theory & $\sigma_{t \bar{t} Z}=0.84 \pm 0.1 \mathrm{pb}$ \\
& $\sigma_{t \bar{t} W}=0.60 \pm 0.07 \mathrm{pb}$ \\
\hline \hline
\end{tabular}

Table 2: Inclusive $t \bar{t} \mathrm{Z}$ and $t \bar{t} \mathrm{~W}$ production cross-section measurements at $13 \mathrm{TeV}$. The theory predictions are computed at NLO QCD precision by MADGRAPH5_aMC@NLO and the uncertainties are primarily due to higher-order corrections.

In addition to the inclusive $t \bar{t}$ production cross-section measurements, ATLAS and CMS have also measured the $t \bar{t}$ production cross-section in association with a vector boson, $W$ or $Z$. These cross-sections may be altered by the presence of physics beyond the SM. The final states used in the measurements contain at least two leptons and some channels up to four leptons. The crosssections are extracted using likelihood fits to the signal and control regions. The $13 \mathrm{TeV}$ ATLAS measurement uses the 2015 dataset $\left(3.2 \mathrm{fb}^{-1}\right.$ ) while the CMS measurement uses the 2015 dataset and some 2016 data $\left(12.9 \mathrm{fb}^{-1}\right)$. The respective results are given in table 2. For the case of the CMS results the statistical significance is $3.9 \sigma(2.6 \sigma)$ observed (expected) for $t \bar{t} W$ and $4.6 \sigma$ (5.8 $\sigma)$ observed (expected) for $t \bar{t} Z$.

For more details on $t \bar{t}$ production cross-section results as well as references for the results presented in this section please see [12].

\subsection{Experimental results on $t \bar{t}$ differential cross-section measurements}

An even more stringent test of QCD calculations than the inclusive $t \bar{t}$ production cross-section is to measure the production cross-section differentially as a function of many different top quark observables. In addition, some models of new physics might distort the differential cross-sections while maintaining the inclusive cross-section within the experimental uncertainties. One such observable is the top quark transverse momentum which has been shown by both ATLAS and CMS to be mismodelled by $\mathrm{MC}$ generators at $7 \mathrm{TeV}$ and $8 \mathrm{TeV}$. The $8 \mathrm{TeV}$ differential cross-section results as a function of the top quark transverse momentum are unfolded to parton level and show a good agreement with NNLO QCD predictions, hinting that the source of the mismodelling in MC generators may be due to missing higher order corrections. It is interesting to probe the NNLO 
QCD calculations in the "boosted" regime, which refers to the phase-space for which the top quarks have a large momentum (greater than about $350 \mathrm{GeV}$ ). At this energy regime, the top quark decay products are collimated into a large (radius $\sim 1.0$ ) jet, necessitating dedicated boosted-jet and toptagging identification techniques. At $13 \mathrm{TeV}$ using the 2015 dataset and some 2016 data, ATLAS measured differential cross-section in the all-hadronic channel where both top quarks are boosted, as shown in figure 1 . Using the $8 \mathrm{TeV}$ dataset, CMS measured, for the first time in the $t \bar{t}$ system, double differential cross-section measurements. As an example of such an application, the double differential measurements involving the rapidity of the $t \bar{t}$ system as well as the invariant mass of the $t \bar{t}$ system give strong constraints on PDF, as shown in figure 1. For more details on $t \bar{t}$ differential production cross-section results as well as references for the results presented in this section please see [13].
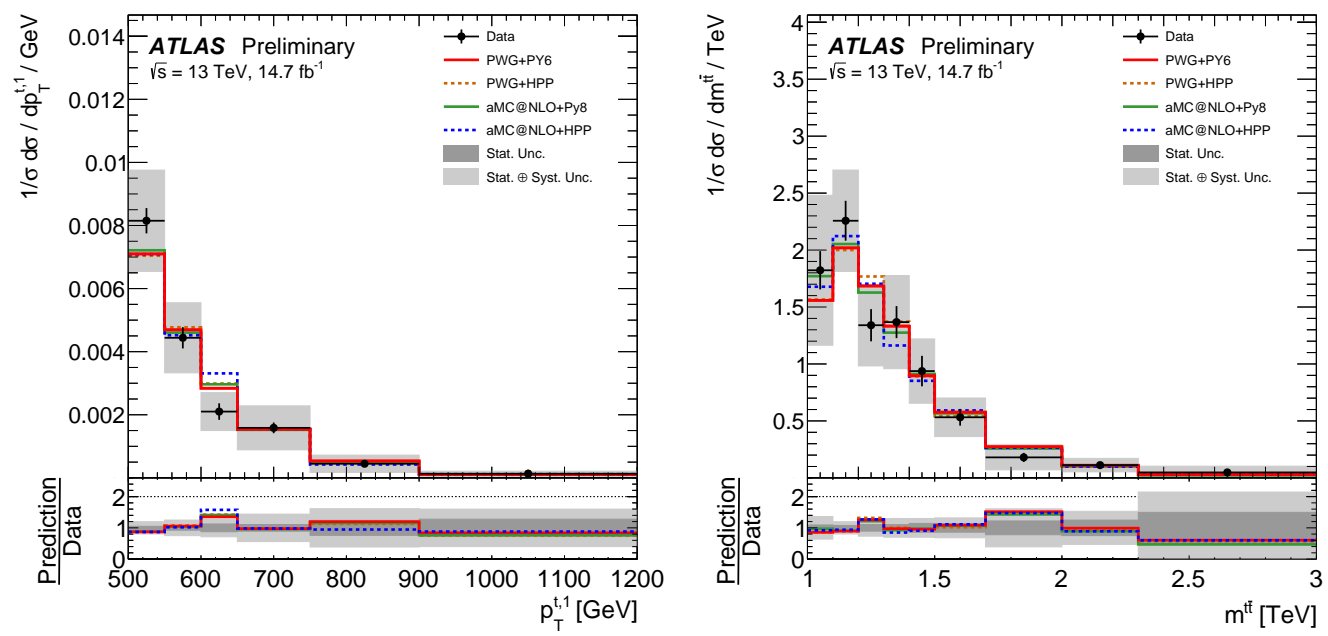

\section{CMS}
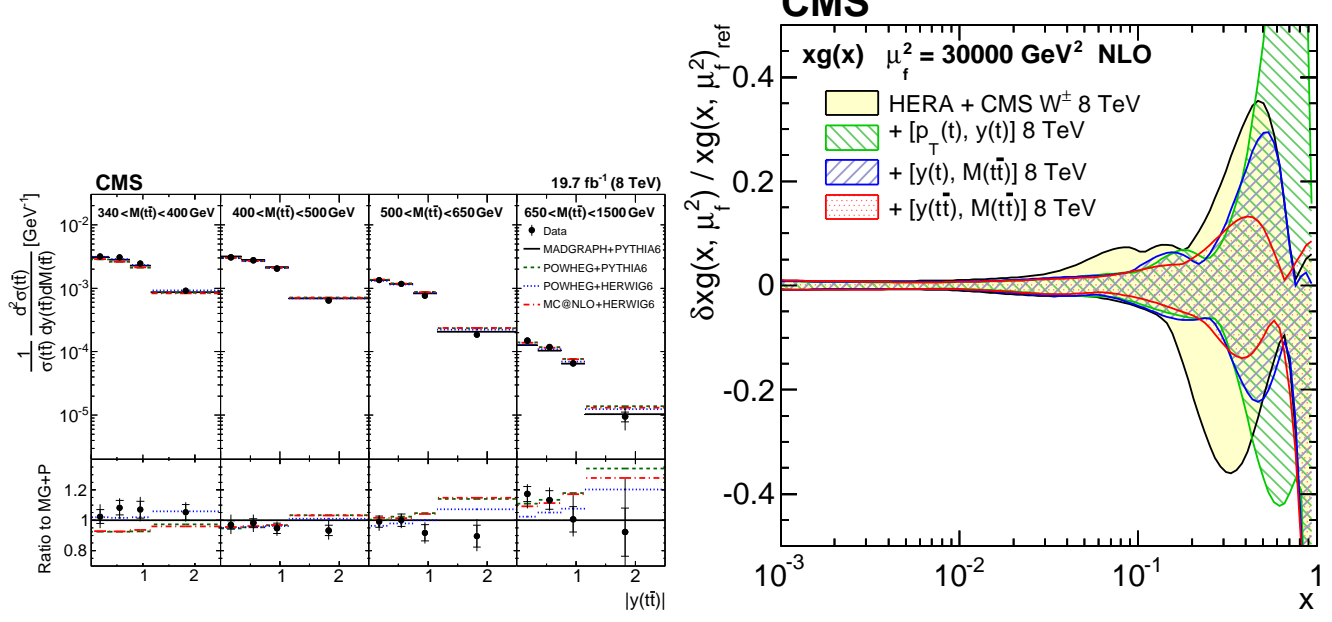

Figure 1: top-left: normalised differential cross-section as a function of the leading boosted top quark particle transverse momentum, top-right: normalised differential cross-section as a function of the invariant mass between the two boosted top quark particles, bottom-left: normalised double-differential cross-section as a function of the $t \bar{t}$ rapidity in regions of invariant mass of the $t \bar{t}$ system, bottom-right: relative total uncertainties of the gluon distribution. 


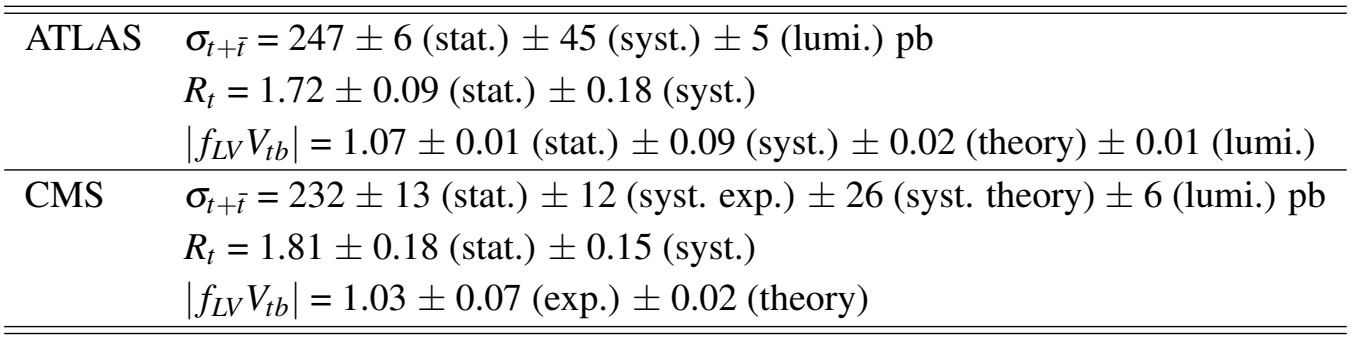

Table 3: Single top quark cross-section in the $t$-channel, as well as $R_{t}$ the ratio of top quark cross-section to anti-top quark and the CKM element $V_{t b}$ where $f_{L V}$ is a left-handed form factor encapsulating non-SM contributions.

\subsection{Experimental results on single top quark production and measurements of $\left|V_{t b}\right|$}

Single top quarks are produced via the electroweak interaction involving different mechanism: the $s$-channel production, the $t$-channel production and the $W t$-channel production. Single top quark measurements can provide direct information on the CKM matrix element $\left|V_{t b}\right|$ since it is involved in both the production and decay of the top quark. These measurements can also be used to search for new particle resonances ( $s$-channel) or for flavour changing neutral currents $(t-$ channel). They also probe the $b$ quark PDF as well as the nature of the $t W b$ coupling. The highest production cross-section is obtained from the $t$-channel (the top quark cross-section is higher than the anti-top quark cross-section, owing to the LHC being a proton-proton machine), amounting to roughly a third of the $t \bar{t}$ production cross-section. Both ATLAS and CMS measure the singletop quark cross-section at $13 \mathrm{TeV}$ using the 2015 dataset requesting one isolated charged lepton (electron or muon) and two jets, one of which is b-tagged. Multi-variate analysis methods are used to discriminate between single top quark $t$-channel events against $t \bar{t}$ and $W+$ jets events, using variables like the top quark mass, the invariant mass of the jet and the b-tagged jet and the pseudorapidity of the light jet. The ratio between the top and anti-top quark cross-section $\left(R_{t}\right)$ as well as $\left|V_{t b}\right|$ are also measured. The results are summarised in table 3.

For more details on single top quark production results as well as references for the results presented in this section please see [14].

\section{Top quark properties and BSM physics}

The large mass of the top quark makes this particle an ideal probe of the electroweak symmetry breaking mechanism and the possible new physics that stabilizes the Higgs boson mass. BSM physics typically includes top quark partners (e.g. the scalar top in SUSY models) and / or other new particles, for instance from an extended Higgs sector.

The tree-level couplings of these new particles to the top quark can have a chiral structure different than the SM couplings, and hence some properties of the top quark can carry the imprint of BSM physics. One important example is the top quark polarization. Since the top quark decays before hadronization, its polarization is amenable to measurement and can be compared to the SM prediction [15]. Possible deviations from SM can be interpreted either in the context of specific theories [16, 17], or in the context of effective field theories (see Sec. 7). 


\subsection{Experimental results on top quark properties}

A measurement of the top quark polarization is done by the CMS experiment using single top quarks produced in the $t$-channel [18]. This analysis uses the $8 \mathrm{TeV}$ dataset but follows a very similar analysis selection as the one described above for the $13 \mathrm{TeV}$ dataset. Due to the $V-A$ coupling structure of the SM, single top quarks are predicted to be produced highly polarized along the direction of the spectator quark, recoiling against the top quark. Physics beyond the SM could lead to a depolarisation of the single top quark at production. The analysis measures the angle between the $W$-muon from the top quark and the recoiling light quark, in the top quark rest frame, $\theta_{\mu}^{*}$. The differential distribution is given by

$$
\frac{1}{\sigma} \frac{d \sigma}{d \cos \theta_{\mu}^{*}}=\frac{1}{2}\left(1+P_{t} \alpha_{\mu} \cos \theta_{\mu}^{*}\right)=\left(\frac{1}{2}+A_{\mu} \cos \theta_{\mu}^{*}\right),
$$

where $P_{t}$ denotes the top quark polarization, $\alpha_{\mu}=1$ in the SM and $A_{\mu}=0.44$ in the SM. The analysis measures a value of $A_{\mu}=0.26 \pm 0.03$ (stat.) \pm 0.10 (syst.), which is about two standard deviations away from the SM prediction.

Measurements of the spin correlation between a top quark and an anti-top quark in $t \bar{t}$ events can also provide information about BSM models. The ATLAS experiment makes use of dilepton events since the polarization-analyzing power of the angular distributions of charged leptons in such events is effectively $100 \%$ [19]. The azimuthal angle $\Delta \phi$ between the charged leptons is used as the observable sensitive to $t \bar{t}$ spin correlation. The data $\Delta \phi$ distribution is fitted to extract a coefficient $f_{S M}$ which measures the degree of spin correlation relative to the SM prediction. A value of $f_{S M}=0$ implies that the top quark spins are uncorrelated while $f_{S M}>1$ would indicate a spin correlation larger than the one predicted by the SM. Using the $8 \mathrm{TeV}$ dataset ATLAS measures a value of $f_{S M}=1.20 \pm 0.05$ (stat.) \pm 0.13 (syst.), which agrees with the SM prediction within two standard deviations. The measurement is also used to search for top squark pair production by fitting each bin of the $\Delta \phi$ distribution, allowing for top squark production within the difference observed between data and the SM prediction. No evidence is found and top squark masses between the top quark mass and $191 \mathrm{GeV}$ are excluded, assuming a $100 \%$ branching ratio for $\tilde{t} \rightarrow t \tilde{\chi}_{1}^{0}$ and $m_{\tilde{\chi}_{1}^{0}}=1 \mathrm{GeV}$.

\subsection{Experimental results on the top quark mass}

The most precise direct measurement of the top quark mass at the Tevatron is performed by D0 using the full dataset $\left(9.7 \mathrm{fb}^{-1}\right)$. The measurement relies on the lepton+jets channel the insitu calibration of the Jet Energy Scale (JES). The results are shown in table 4 and correspond to a $0.43 \%$ uncertainty on the top quark mass. The dominant systematic uncertainties are the hadronization and underlying event, the residual JES and the $b$-quark JES. The CMS experiment also uses the lepton+jets channel to measure the most precise top quark mass at the LHC, using the $8 \mathrm{TeV}$ dataset. The analysis combines both the in-situ JES approach and standard JES constraints to produce a "hybrid" measurement. The results are shown in table 4 showing a $0.3 \%$ uncertainty. The dominant uncertainty is the $b$-quark JES at the level of $0.32 \mathrm{GeV}$.

Direct top mass measurements suffer from the interpretation of this parameter in terms of higher-order corrections. A theoretically cleaner concept is that of the top-quark "pole mass". Such 


\begin{tabular}{ll}
\hline \hline D0 & $m_{t}=174.98 \pm 0.58$ (stat. + JES) \pm 0.49 (syst.) $\mathrm{GeV}$ \\
CMS & $m_{t}=172.35 \pm 0.16$ (stat. + JES) \pm 0.48 (syst.) GeV \\
\hline \hline
\end{tabular}

Table 4: Direct top quark mass measurements from the D0 and CMS experiments.

\begin{tabular}{ll}
\hline \hline CMS & $m_{t}^{\text {pole }}=176.7 \pm 2.9 \mathrm{GeV}$ \\
ATLAS & $m_{t}^{\text {pole }}=173.7 \pm 2.2 \mathrm{GeV}$ \\
D0 (NLO) & $m_{t}^{\text {pole }}=167.3 \pm 2.6 \mathrm{GeV}$ \\
D0 (NNLO) & $m_{t}^{\text {pole }}=169.1 \pm 2.5 \mathrm{GeV}$ \\
\hline \hline
\end{tabular}

Table 5: Top quark pole mass measurements from the CMS, ATLAS and D0 experiments.

measurements make use for example of the $t \bar{t}$ production cross-section and its dependence on the top quark mass. The current uncertainty on such top quark pole mass are of the order of $1.5 \%$. The CMS experiment performs this measurement using the 2015 dataset at $13 \mathrm{TeV}$ in the lepton+jets channel and making use of the inclusive $t \bar{t}$ cross-section measurement. The ATLAS experiment utilizes the $\sigma_{t \bar{t}+1 \text { jet }}$ since the radiation rate of a high- $p_{T}$ gluon off the $t \bar{t}$ system is proportional to the top quark pole mass. The $7 \mathrm{TeV}$ lepton+jets dataset is used by the ATLAS measurement. The D0 experiment extracts the top quark pole mass using differential measurements of the $t \bar{t}$ crosssections as a function of the $t \bar{t}$ invariant mass and the top-quark $p_{T}$. Table 5 summarizes these top quark pole mass measurements.

For more details on measurements of the top quark mass as well as references for the results presented in this section please see [20].

\section{Top quark charge asymmetries}

The measurement of charge asymmetries provides another tool to search for non-standard physics. At a proton-antiproton collider, the charge asymmetry manifests itself as a forwardbackward asymmetry $\left(\mathscr{A}_{\mathrm{FB}}\right)$, with a SM expectation of the order of $10 \%$, while for proton-proton collisions at the LHC the observable rapidity asymmetry is an order of magnitude smaller [21]. Higher-order corrections are also known [22, 23, 24].

The measurement of a large $\mathscr{A}_{\mathrm{FB}}$ at the Tevatron has triggered quite some activity in BSM model building to explain the effect (see Ref. [25] for a review). Although the original large measurement is by now superseded (see Section 4.1), it has established the charge asymmetries as an important observable sensitive to new physics. On the other hand, there is no forward-backward asymmetry at the LHC due to the symmetric (proton-proton) initial state. Other observables that are sensitive to the underlying charge asymmetry have been devised, see [26, 27] for recent ideas. For a more comprehensive overview, please see [28].

\subsection{Experimental results on top-quark-related asymmetries}

At the Tevatron the forward-backward asymmetry of the $t \bar{t}$ production is predicted comparing the rapidity of the top quark with the one from the anti-top quark. Using the full dataset collected by $\mathrm{CDF}$ and D0, the experiments measure an inclusive forward-backward asymmetry that is compatible with the latest theory calculation to within approximately one standard deviation. 
The ATLAS experiment has recently measured the $t \bar{t}$ charge asymmetry using the $8 \mathrm{TeV}$ dataset in the dilepton channel. Both inclusive asymmetries and differential ones (as a function of the invariant mass, transverse momentum and boost of the $t \bar{t}$ system) are measured using two observables: $A_{C}^{\ell \ell}$ which uses the selected leptons and $A_{C}^{t \bar{t}}$ which uses the reconstructed $t \bar{t}$ system. The inclusive asymmetries are measured in the full phase-space to be: $A_{C}^{\ell \ell}=0.008 \pm 0.006$ and $A_{C}^{t \bar{t}}$ $=0.021 \pm 0.016$, in agreement with the SM predictions.

The CMS experiment has performed a novel measurement using $t \bar{t}$ events in the lepton+jets channel to test for CP-violation using the $8 \mathrm{TeV}$ dataset. The event selection is similar to the measurement from CMS of the $t \bar{t}$ charge asymmetry in the same channel. CP-violation is tested by building asymmetries involving various T-odd triple products of the final state objects momenta. The measured asymmetries show no evidence for CP-violation.

The ATLAS experiment also performed a novel CP-violation measurement using $t \bar{t}$ lepton+jets events and the $8 \mathrm{TeV}$ dataset. The measurement aims to test CP-violation in $b$-decays, where the $b$ quarks have come from the decay of the top and anti-top quarks. Asymmetries are built comparing the charge of the $W$-lepton (b-quark charge at production) to the charge of a muon coming from the semileptonic decay of a $b$-hadron ( $b$-quark charge at decay). Two assymmetries are measured (Same Sign and Opposite Sign ones) that are sensitive to direct CP-violation and $\mathrm{CP}$-violation in mixing. The results obtained are consistent with the SM and are dominated by statistical uncertainties. The measurements include the first limit for direct CP-violation in the $b$ to $c$ decay: $A_{\text {dir }}^{b c}=-0.010 \pm 0.011$ and the strongest limit on direct CP-violation in the $c$ to $\ell$ decay: $A_{\text {dir }}^{c \ell}=0.009 \pm 0.010$.

For more details on measurements of top-quark-related asymmetries as well as references for the results presented in this section please see [29].

\section{Results on the interaction between the Higgs boson and the top quark}

Being the heaviest particle, the top quark has the strongest Yukawa coupling with the Higgs boson. Measuring this Yukawa coupling is one of the highest priority for the ATLAS and CMS experiments. Although the top Yukawa coupling is involved in gluon-fusion Higgs production as well as the Higgs decay into a pair of photons via one-loop diagrams, measuring the cross-section of a Higgs boson in association with a $t \bar{t}$ pair is a crucial and complementary way to measure this coupling in a tree-level process.

Due to the different $t \bar{t}$ channels combined with the various possible Higgs decay products, many $t \bar{t} H$ analyses are performed. Already with $13 \mathrm{fb}^{-1}$ of $13 \mathrm{TeV}$ data, the precision of the measurements has reached the level achieved with the full $8 \mathrm{TeV}$ dataset. A summary of the results for the ratio of the measured $t \bar{t} H$ cross-section to that of the one predicted by the SM, $\mu$, is presented in table 6.

\section{Physics beyond the SM searches using top quarks}

Searching for new physics with top quarks involves direct searches for new resonances or decays of exotic top partners, as well as searches for rare top-quark decay modes. 


\begin{tabular}{ll}
\hline \hline$t \bar{t} H(b \bar{b})$ combined dilepton and lepton+jets ATLAS & $\mu=2.1_{-0.9}^{+1.0}$ \\
$t \bar{t} H(b \bar{b})$ combined dilepton and lepton+jets CMS & $\mu=-0.19 \pm 1.1$ \\
$t \bar{t} H(\gamma \gamma)$ ATLAS & $\mu=-0.25_{-0.99}^{+1.26}$ \\
$t \bar{t} H(\gamma \gamma)$ CMS & $\mu=1.91_{-1.2}^{+1.5}$ \\
\hline$t \bar{t} H 13$ TeV combination ATLAS & $\mu=1.8 \pm 0.7$ \\
$t \bar{t} H 7,8$ and 13 TeV combination ATLAS & $\mu=1.7 \pm 0.8$ \\
\hline \hline
\end{tabular}

Table 6: Best fit for the ratio of the measured $t \bar{t} H$ cross-section to that of the one predicted by the SM using $m_{H}=125 \mathrm{GeV}[30,31,32,33,34]$.

The precise measurement of rare decay modes as a tool to probe new physics has a long tradition in $B$ physics. With the large number of top quarks produced at the LHC, this strategy can also be applied in the top sector.

For instance, flavour changing neutral current (FCNC) processes change the flavour of a fermion current without altering its electric charge. In the case of top quarks this corresponds to: $t \rightarrow u / c+\gamma / Z / g / H$ (in either top production or top decay). FCNC are suppressed in the SM by the GIM mechanism. Due to the light masses of the down-type quarks, this effect is even more pronounced in top vs. $B$-meson decays, such that the SM contribution to these observables is beyond the senisitivity of the LHC experiments. (For instance, the mode $t \rightarrow c g$ has the largest SM branching ratio, of the order of $10^{-12}$.) This makes FCNC top quark decays a clean probe of new physics. Many BSM models predict new contributions to FCNC in the top-quark sector that are orders of magnitude larger than the SM predictions, and are not yet excluded from $B$-physics observables [35].

\subsection{Experimental results on searches for flavour changing neutral currents}

A recent result from the CMS experiment uses the $8 \mathrm{TeV}$ dataset and looks for FCNC in topquark production: $t \rightarrow u / c+\gamma$. The analysis searches for events containing a single isolated muon, a single isolated photon, some transverse missing energy and one $b$-tagged jet. The dominant background is the $W+\gamma$ production and a Boosted Decision Tree is used to discriminate between the signal and backgrounds. The $95 \%$ confidence level branching ratio limit obtained is: $1.3 \times$ $10^{-4}\left(1.7 \times 10^{-3}\right)$ for $t \rightarrow u(c)+\gamma$. The dominant uncertainty is from the data-driven background estimation.

A recent result from the ATLAS experiment uses the $8 \mathrm{TeV}$ dataset and looks for FCNC in top-quark decay using $t \bar{t}$ events: $t \rightarrow u / c+Z$. The analysis searches for events containing three isolated electrons or muons, at least two jets, some missing transverse energy and one $b$-tagged jet. The backgrounds include diboson production as well as $t \bar{t}$ events produced in association with a vector boson. The obtained $95 \%$ confidence level branching ratio limit is $7 \times 10^{-4}$.

Overall the searches for FCNC in the top-quark sector are starting to approach the branching ratios predicted by new physics models, for example for 2-Higgs doublet models with flavour violation. For more details on FCNC in the top quark sector as well as references for the results presented in this section please see [36]. 


\subsection{Experimental results on resonance searches}

Many BSM models predict high mass particles decaying to $t \bar{t}$ events and experiments have actively searched for these and have produced limits of various models ((Technicolour $Z^{\prime}$, KaluzaKlein gravitons or gluons, heavy Higgs bosons, etc.). In general, the interference produced between those processes and the SM processes giving the same final states has not been modelled. Recently, some earlier limits have been revisited by ATLAS using the $8 \mathrm{TeV}$ dataset in order to take into account those interference effects. For example, new limits are obtained for $g g \rightarrow A(H) \rightarrow t \bar{t}$ of $\tan \beta<0.85(0.45)$ for $m_{A / H}=500 \mathrm{GeV}$ [37].

As the mass of the new particle increases, the decayed top quarks get more and more boosted and the decay products get merged into a single large jet, necessitating dedicated reconstruction methods, as mentioned earlier in Section 2.2. Both the CMS and ATLAS experiments have measured heavy resonance searches at $13 \mathrm{TeV}$ using the 2015 dataset $[38,39,40]$. The strongest limit is obtained by the CMS experiment using the $8 \mathrm{TeV}$ dataset and combining the dilepton, lepton+jets and all-hadronic channels. The $95 \%$ confidence limit on the mass of a $Z^{\prime}$ with a $1 \%$ width is $M_{Z^{\prime}}$ $<2.4 \mathrm{TeV}$, and the limit on the mass of a Randall-Sundrum Kaluza-Klein gluon is $M_{g}<2.7 \mathrm{TeV}$ (with $\sigma_{g}$ going from $17 \mathrm{pb}$ to $0.059 \mathrm{pb}$ for $M_{g}$ going from $0.7 \mathrm{TeV}$ to $3 \mathrm{TeV}$ ).

\subsection{Experimental results on other new physics searches}

In Little Higgs and Composite Higgs models Vector-Like Quarks (VLQs) are predicted and expected to couple preferentially to third-generation quarks. A sufficiently heavy up-type $T$ quark can decay not only to a $W$ and $b$-quark, but also to a Higgs or a $Z$ and a top quark: $T \rightarrow W b, Z t, H t$. Similarly a down-type $B$ quark can decay into a Higgs or $Z$ and a $b$-quark, in addition to a $W$ and top quark: $B \rightarrow W t, Z b, H b$. The ATLAS and CMS experiments actively look for such VLQs. The ATLAS experiment obtains limits on the $T$ and $B$ masses by making use of the $8 \mathrm{TeV}$ dataset in the $t \bar{t}$ lepton+jets channel, looking for the decay channels that involve the $W$ and the Higgs boson $(b \bar{b}$ final state). Taking into account previous searches from ATLAS that involved multi-lepton final states, the final observed lower limits on the $T$ quark mass range between $730 \mathrm{GeV}$ and $950 \mathrm{GeV}$ for all possible values of the branching ratios into the three decay modes. Similar limits for the $B$ quark mass range between $575 \mathrm{GeV}$ and $813 \mathrm{GeV}$.

The CMS experiment also performs a search for VLQ $T$ quarks using the $13 \mathrm{TeV}$ dataset collected in 2015. The event selection requires at least one lepton in the final state and treats the branching ratios of the three decay modes as free parameters, although the search is most sensitive to the case of the $T$ quark decaying to a top quark and a Higgs boson. As the focus is on high mass VLQ, the search employs boosted reconstruction techniques to look for the Higgs decay into a pair of $b$ quarks. Upper 95\% confidence level exclusion limits on $T$ quark pairs cross-sections are calculated for multiple branching ratio scenarios. Assuming $100 \%$ for the $T \rightarrow H t, T$ quark masses below $860 \mathrm{GeV}$ are excluded.

For more details on new physics searches in the top quark sector as well as references for the results presented in this section please see [41].

\section{Effective field theory - collider constraints}

The consistency of the measurements at the LHC with the predictions of the standard model 
can be interpreted as a hint towards a scale of new physics that is higher than the currently available maximal center-of-mass energy. In this situation, an EFT approach [42, 43] allows to describe new phenomena in a very general setup, comprising a wide class of UV completions in a unified framework. Whereas only a handful of operators are relevant for top-quark physics, there are many observables being measured, for instance, single top and top quark pair production total and differential cross sections, charge asymmetries, and $W$ helicity fractions. This allows for a global fit of all top quark operators [44, 45, 46]. However, current limits are generally poor and not yet helpful. Apart from collecting more statistics, the sensitivity can be improved by looking at the tails of differential distributions. For more details, please see [47].

\section{Effective field theory - precision constraints}

Compared to collider observables, precision probes lead to complementary and, in several occasions, more stringent constraints on new interactions. This is in particular the case for imaginary top-Higgs couplings that can be tested in CP-violating observables like electric dipole moments [48, 49, 50, 51].

Focusing on the $\mathrm{CP}$ violating dimension-six operators in the top Higgs sector, a systematic analysis of precision constraints can be performed, with constraints coming mainly from the electric dipole moments of the neutron and the electron, where the SM background is several orders of magnitude below the current limits [54]. This analysis involves the renormalisation-group evolution from scales above electroweak symmetry breaking down to the hadronic scale of order $1 \mathrm{GeV}$. QCD running and higher-order matching corrections can have a large impact on the phenomenology and existing limits can be improved by up to three orders of magnitude [53].

One major issue with bounds derived from electric dipole moments of hadronic systems is the large theoretical uncertainty in the requisite hadronic matrix elements (see, e.g., [54]). It is important to systematically account for these uncertainties $[52,53]$ in a global fit, where associated cancellations can considerably weaken the bounds by orders of magnitude. See [55] for more details. For a concrete example leading to top quark FCNC transistions, see [56].

\section{Conclusions}

Testing the flavor structure of the SM in high- $p_{T}$ processes is an important field of research. It is made possible by the direct production at the LHC of the heaviest SM particles, the Higgs boson and the top quark. This working group has focused on studying top and Higgs production and decay directly on the one hand, and the complementarity of observables in the Higgs and top sector of the SM to those in $B, D$, and $K$ decays as well as further precision probes like electric dipole moments, on the other hand.

\section{References}

[1] M. Czakon, P. Fiedler and A. Mitov, Phys. Rev. Lett. 110 (2013) 252004

[2] P. Bärnreuther, M. Czakon and A. Mitov, Phys. Rev. Lett. 109 (2012) 132001

[3] M. Czakon, D. Heymes and A. Mitov, Phys. Rev. Lett. 116 (2016) 082003 
[4] M. Czakon, P. Fiedler, D. Heymes and A. Mitov, JHEP 1605 (2016) 034

[5] P. Mathews, "SM $t \bar{t}$ cross section", in proceedings of "9th International Workshop on the CKM Unitarity Triangle" PoS(CKM2016)116

[6] R. Aaij et al. [LHCb Collaboration], Phys. Lett. B 767 (2017) 110

[7] M. Czakon and A. Mitov, Comput. Phys. Commun. 185 (2014) 2930

[8] M. Cacciari, M. Czakon, M. Mangano, A. Mitov and P. Nason, Phys. Lett. B 710 (2012) 612

[9] M. Beneke, P. Falgari, S. Klein and C. Schwinn, Nucl. Phys. B 855 (2012) 695

[10] M. Czakon and A. Mitov, JHEP 1212 (2012) 054

[11] M. Czakon and A. Mitov, JHEP 1301 (2013) 080

[12] A. Nayak, "Recent inclusive $t \bar{t}$ cross section measurements", in proceedings of "9th International Workshop on the CKM Unitarity Triangle" PoS(CKM2016)117

[13] R. di Sipio, "LHC and Tevatron results on $t \bar{t}$ differential cross sections", in proceedings of "9th International Workshop on the CKM Unitarity Triangle" PoS(CKM2016)118

[14] S. Mitra, "Single top and Vtb measurements", in proceedings of "9th International Workshop on the CKM Unitarity Triangle" PoS(CKM2016)119

[15] W. Bernreuther, A. Brandenburg, Z. G. Si and P. Uwer, Nucl. Phys. B 690 (2004) 81

[16] W. Bernreuther, P. Gonzalez and M. Wiebusch, Eur. Phys. J. C 60 (2009) 197

[17] L. Duarte, G. A. GonzÃąlez-Sprinberg and J. Vidal, JHEP 1311 (2013) 114

[18] CMS Collaboration, JHEP 04 (2016) 073

[19] ATLAS Collaboration, PRL 114 (2015) 142001

[20] O. Brandt, "Measurements of the top quark mass from the LHC and the Tevatron" in proceedings of "9th International Workshop on the CKM Unitarity Triangle" PoS(CKM2016)122

[21] J. H. Kühn and G. Rodrigo, Phys. Rev. D 59 (1999) 054017

[22] V. Ahrens, A. Ferroglia, M. Neubert, B. D. Pecjak and L. L. Yang, JHEP 1109 (2011) 070

[23] V. Ahrens, A. Ferroglia, M. Neubert, B. D. Pecjak and L. L. Yang, Phys. Rev. D 84 (2011) 074004

[24] W. Hollik and D. Pagani, Phys. Rev. D 84 (2011) 093003

[25] J. F. Kamenik, J. Shu and J. Zupan, Eur. Phys. J. C 72 (2012) 2102

[26] S. Berge and S. Westhoff, JHEP 1307 (2013) 179

[27] S. Berge and S. Westhoff, Phys. Rev. D 95 (2017) 014035

[28] S. Westhoff, "Review of top charge asymmetries for LHC run II", in proceedings of "9th International Workshop on the CKM Unitarity Triangle" PoS(CKM2016)120

[29] J. Morris, "Asymmetries in the top sector", in proceedings of "9th International Workshop on the CKM Unitarity Triangle" PoS(CKM2016)124

[30] ATLAS Collaboration, ATLAS-CONF-2016-080

[31] ATLAS Collaboration, ATLAS-CONF-2016-067

[32] ATLAS Collaboration, ATLAS-CONF-2016-068 
[33] CMS Collaboration, CMS-PAS-HIG-16-038

[34] CMS Collaboration, CMS-PAS-HIG-16-020

[35] P. J. Fox, Z. Ligeti, M. Papucci, G. Perez and M. D. Schwartz, Phys. Rev. D 78 (2008) 054008

[36] S. Bhomik, "Flavour Changing Neutral Current searches in the top quark sector" in proceedings of "9th International Workshop on the CKM Unitarity Triangle" PoS(CKM2016)126

[37] ATLAS Collaboration, ATLAS-CONF-2016-073

[38] ATLAS Collaboration, ATLAS-CONF-2016-014

[39] CMS Collaboration, CMS-PAS-B2G-15-002

[40] CMS Collaboration, CMS-PAS-B2G-15-003

[41] P. Das, "New Physics Searches in the Top Quark Sector in Hadron Colliders" in proceedings of "9th International Workshop on the CKM Unitarity Triangle" PoS(CKM2016)129

[42] W. Buchmuller and D. Wyler, Nucl. Phys. B 268 (1986) 621.

[43] B. Grzadkowski, M. Iskrzynski, M. Misiak and J. Rosiek, JHEP 1010 (2010) 085

[44] A. Buckley, C. Englert, J. Ferrando, D. J. Miller, L. Moore, M. Russell and C. D. White, Phys. Rev. D 92 (2015) 091501

[45] A. Buckley, C. Englert, J. Ferrando, D. J. Miller, L. Moore, M. Russell and C. D. White, JHEP 1604 (2016) 015

[46] C. Englert, L. Moore, K. Nordström and M. Russell, Phys. Lett. B 763 (2016) 9

[47] A. Buckley et al., "Results from TopFitter", in proceedings of "9th International Workshop on the CKM Unitarity Triangle" PoS(CKM2016)127

[48] A. Cordero-Cid, J. M. Hernandez, G. Tavares-Velasco and J. J. Toscano, J. Phys. G 35 (2008) 025004

[49] J. F. Kamenik, M. Papucci and A. Weiler, Phys. Rev. D 85 (2012) 071501 Erratum: [Phys. Rev. D 88 (2013) 039903]

[50] J. Brod, U. Haisch and J. Zupan, JHEP 1311 (2013) 180

[51] M. Gorbahn and U. Haisch, JHEP 1406 (2014) 033

[52] V. Cirigliano, W. Dekens, J. de Vries and E. Mereghetti, Phys. Rev. D 94 (2016) 034031

[53] V. Cirigliano, W. Dekens, J. de Vries and E. Mereghetti, Phys. Rev. D 94 (2016) 016002

[54] M. Pospelov and A. Ritz, Annals Phys. 318 (2005) 119

[55] W. Dekens, "High- and low-energy constraints on top-Higgs couplings", in proceedings of "9th International Workshop on the CKM Unitarity Triangle" PoS(CKM2016)125

[56] D. Bardhan, "FCNC Decays of the Top Quark", in proceedings of "9th International Workshop on the CKM Unitarity Triangle" PoS(CKM2016)131 